\title{
Inhibiting Rho kinase 2 reduces memory dysfunction in adult rats exposed to sevoflurane at postnatal days 7-9
}

\author{
TAO HAN, ZHONGHUA HU, YONGZHONG TANG, ALISHA SHRESTHA, WEN OUYANG and QIN LIAO
}

\begin{abstract}
Department of Anesthesiology, The Third Xiangya Hospital, Central South University, Changsha, Hunan 410013, P.R. China
\end{abstract}
Received October 24, 2014; Accepted February 6, 2015

DOI: $10.3892 /$ br. 2015.429

\begin{abstract}
The aim of the present study was to investigate the roles of Rho protein A (RhoA) and Rho kinases 2 (ROCK2) in the memory dysfunction of adult rats exposed to sevoflurane at postnatal days 7-9 (P7-9). One-week-old Sprague-Dawley rats were divided into four groups known as C, S1, S3 and F. Rats in the $\mathrm{S} 1$ (2 $\mathrm{h}$ at $\mathrm{P} 7)$ and $\mathrm{S} 3$ groups ( $2 \mathrm{~h} / \mathrm{day}$ at $\mathrm{P} 7-9)$ were exposed to sevoflurane. The rats in the $\mathrm{F}$ group were treated with the ROCK2 inhibitor and subsequent sevoflurane exposure (2 h/day at $\mathrm{P} 7-9)$. The rats in the $\mathrm{C}$ group received no sevoflurane. The protein levels of RhoA, ROCK 2 and cleaved caspase-3 (Cl-Csp3) in the adult hippocampus were assessed by western blot analysis. Learning and memory of rats at postnatal 45-50 days (P45-50) were detected by the Morris water maze (MWM) test. During the training of MWM, the latency and distance of rats in the $\mathrm{S} 3$ group were significantly longer than that of the $\mathrm{C}$ group $(\mathrm{P}<0.05$, respectively). In the probe test, the percentages of time and distance in the target quadrant for the S3 group were evidently less than that of the $\mathrm{C}$ group $(\mathrm{P}<0.05)$. There was no significant difference in the behaviors between the $\mathrm{C}$ and $\mathrm{S} 1$ groups $(\mathrm{P}>0.05$, respectively). Corresponding to the behavioral changes, the levels of RhoA, ROCK2 and Cl-Csp3 in the hippocampus of the S3 group significantly increased, compared to that of the $\mathrm{C}$ and $\mathrm{S} 1$ groups $(\mathrm{P}<0.05)$. Additionally, the ROCK2 inhibitor clearly decreased ROCK2 and Cl-Csp3 expression and shortened the latency during the training $(\mathrm{P}<0.05, \mathrm{P} 46-49$ respectively) and probe test $(\mathrm{P}<0.05)$ in the $\mathrm{F}$ group, compared to that of the $\mathrm{S} 3$ group. Compared to the $\mathrm{C}$ group, the expression of RhoA, ROCK2 and Cl-Csp3 in the hippocampus of the $\mathrm{S} 1$ group had no significant difference $(\mathrm{P}>0.05)$. Multiple inhalation of sevoflurane can induce neurotoxicity and memory dysfunction. RhoA and ROCK 2 played important roles in the impairment of learning and memory of adults rats exposed to sevoflurane at the postnatal early stage.
\end{abstract}

Correspondence to: Dr Qin Liao, Department of Anesthesiology, The Third Xiangya Hospital, Central South University, 138 Tongzipo Road, Changsha, Hunan 410013, P.R. China

E-mail: xy3yyliaoqin@sina.com

Key words: Rho kinase 2, cognitive dysfunction, sevoflurane, fasudil hydrochloride, neurotoxicity

\section{Introduction}

Pediatric patients may be at risk for later learning and behavioral impairment when exposed to general anesthesia and surgery (1-4). This is consistent with studies in preclinical experiments by Yon et al (5). In the study, rats were exposed at postnatal day 7 to compound anesthetic agents (midazolam, isoflurane and nitrous oxide) and identified neuronal apoptosis in the brain and persistent defects of memory and learning when the rats were juvenile. In addition, these rats also showed the impairment of spatial reference and working memory as they grew up. Similar impairment of learning and memory induced by neonatal anesthesia was also reported for adult patients and other animals (1-4). However, the exact mechanism underlying the neonatal anesthesia remains unclear.

Rho kinase 2 (ROCK2), one of the members of the family of serine/threonine protein kinase, is a downstream effector of Rho protein A (RhoA) (6). RhoA regulates the reorganization of cytoskeleton protein actin by controlling ROCK2, affecting cell migration, apoptosis, gene transcription, nerve regeneration and other biological processes $(7,8)$. RhoA/Rho-kinase is an important pathway underlying neuronal injury in rats of experimental spinal cord injury (9). In addition, the Rho/Rho-kinase pathway is associated with the modulation of $N$-methyl-D-aspartate (NMDA) receptor function and the NMDA receptor is associated with learning and memory processing (10). In a previous study, Lemkuil et al (11) demonstrated that isoflurane induced neurotoxicity of mouse neurons by activating $\mathrm{p} 75^{\mathrm{NTR}}-\mathrm{RhoA}$, and inhibiting activation of RhoA attenuated isoflurane-induced impairment. Pearn et al (12) also reported that propofol-induced apoptosis is involved in $\mathrm{p} 75^{\mathrm{NTR}}$ and RhoA kinase activation in developing neurons in vivo and in vitro. These suggested that RhoA played important roles in the neurotoxicity of anesthetics. However, it remains unclear whether ROCK2, the key downstream molecule of the RhoA signals, mediates the neurotoxicity of anesthetics cognitive impairment of adult rats with neonatal exposure of sevoflurane.

The present study investigated i) the effects of sevoflurane exposure of rats at postnatal days 7-9 on their learning and memory at adulthood; and ii) the association of the cognitive dysfunction induced by sevoflurane to expression changes of RhoA and ROCK2. Sevoflurane exposure at postnatal days 7-9 impaired the learning and memory of rats after they grew up. Corresponding to the behavioral changes of rats, hippocampal RhoA and ROCK2 increased; and inhibiting ROCK2 
expression reversed the cognitive dysfunction induced by sevoflurane exposure.

\section{Materials and methods}

Animals. Fifty-six Sprague-Dawley rats (7 days old) were obtained from the Experimental Animal Center of The Third Xiangya Hospital of Central South University (Changsha, China). The institutional guidelines regarding animal safety were strictly followed and authorized by the Institutional Animal Care. The room temperature was maintained at $22-24^{\circ} \mathrm{C}$ under suitable humidity. The rats were divided into four groups (S1, S3, F and C) randomly, with 14 in each group. The rats in group S1 inhaled 2\% sevoflurane and $80 \%$ oxygen for $2 \mathrm{~h}$. Rats in the S3 group were exposed to sevoflurane three times from postnatal day 7 ( $2 \mathrm{~h}$ /time/day). The F group rats were pretreated with ROCK2 inhibitor fasudil hydrochloride (10 mg/kg, HY-10341; MedChem Express LLC, Princeton, NJ, USA) and $2 \mathrm{~h}$ later were exposed to sevoflurane three times from postnatal day 7 ( $2 \mathrm{~h} /$ time/day). The rats in group C inhaled $80 \%$ oxygen. All the rats were placed in a self-made organic glass box $(30 \times 40 \times 30 \mathrm{~cm})$ with soda lime in the bottom. The multi-function monitor (Datex-Ohmeda, Helsinki, Finland) was used to monitor the concentration of sevoflurane, $\mathrm{O}_{2}$ and $\mathrm{CO}_{2}$. When experimental models were completed for $3 \mathrm{~h}$, rats were sacrificed by decapitation and the hippocampus was removed to assess the expression of RhoA, ROCK2 and cleaved caspase-3 (Cl-Csp3) by western blotting. The neurobehavioral tests of the Morris water maze (MWM) at P45-50 were used to examine the memory function. Latency to locate the target platform, swimming distance and speed in target zone were determined and compared in all the groups.

Western blotting. The protein concentration of samples was determined using a bicinchoninic acid protein assay kit (Wellbio, Changsha, China) according to the manufacturer's instructions. Equal amounts of protein samples (/lane) were loaded and separated by sodium dodecyl sulfate-polyacrylamide gel electrophoresis and transferred to polyvinylidene fluoride membranes. Membranes were blocked with $10 \%$ skimmed milk in phosphate-buffered saline with Tween-20 buffer for $2 \mathrm{~h}$ and subsequently incubated with primary antibodies [rabbit anti-RhoA polyclonal antibody (1:600; cat. no. 10749-1-AP; Proteintech, Wuhan, Hubei, China), rabbit anti-ROCK2 polyclonal antibody, 1:200; cat. no. sc5561; rabbit anti-cleaved caspase3 polyclonal antibody (1:400; cat. no. sc22139; Santa Cruz Biotechnology, Santa Cruz, CA, USA) and anti- $\beta$-actin (1:2,000; cat. no. KGAA001-4; KeyGen BrioTech, Nanjing, China) overnight at $4^{\circ} \mathrm{C}$. After three washes, membranes were incubated with the secondary antibody [goat anti-rabbit IgG (H+L) (1:2,000; cat. no. KGAA35; KeyGen BioTech)] at room temperature for $2 \mathrm{~h}$. Finally, visualization of the proteins was accomplished by ECL detection reagents (Advansta Corp., Menlo Park, CA, USA). The images were developed on luminescent image analyzer (ImageQuant 350 Capture; GE Healthcare Life Sciences, Shanghai, China) and quantified by densitometry (Beckman Coulter, Inc., Pasadena, CA, USA). Relative expression levels of protein were normalized by the ratio of the target protein (ROCK2, RhoA and Cl-Csp3) to $\beta$-actin.
MWM test. The MWM test was used to evaluate the learning and memory of rats. A computerized video track system (Logitech, Suzhou, China) was used to record the movement of the rats in the water maze by following a previous method (13). Briefly, a transparent circular platform was placed below the water surface of the southeast quadrant in a circular black pool. During the training, rats were first placed on the platform for $30 \mathrm{sec}$, and subsequently were put into the water facing the tank wall. The maximum trial time was $60 \mathrm{sec}$, following a relaxation of $20 \mathrm{sec}$ on the platform. When a rat could not locate the platform within $60 \mathrm{sec}$, it was guided to the platform and remained there for $30 \mathrm{sec}$. All the rats were trained for 5 days with three trials/day. Following training, the memory of the rats was evaluated by the percentage of searching time and distance in the targeted area.

Statistical analysis. Water maze data are presented as mean \pm standard error of the mean and were analyzed using analysis of variance for repeated measures followed by the least significant difference test. Western blotting data are presented as mean \pm standard deviation and were analyzed using a Student's t-test. $\mathrm{P}<0.05$ was considered to indicate a statistically significant difference.

\section{Results}

Sevoflurane exposure at postnatal day 7-9 impairs the learning and memory of rats after they grew up. In the training, the latency and distance of rats in the S3 group at postnatal days 45-50 (P45-50) were significantly longer than that of the $\mathrm{C}$ group $(\mathrm{P}<0.05$, respectively). There was no significant difference of the latency and distance between the $\mathrm{C}$ and $\mathrm{S} 1$ groups ( $\mathrm{P}>0.05$, respectively) (Fig. $1 \mathrm{~A}$ and $\mathrm{B})$. In all the groups, the swimming speed did not show a significant difference on all the days ( $\mathrm{P}>0.05)$ (Fig. 1C). In the probe trial test, the percentage of searching time and distance in target quadrant in the $\mathrm{S} 3$ group was evidently less than that of the $\mathrm{C}(\mathrm{P}<0.05)$ and $\mathrm{S} 1$ groups $(\mathrm{P}<0.05)$. The percentage of searching time and distance in target quadrant in the $\mathrm{S} 1$ group were not different from that of the $\mathrm{C}$ group ( $\mathrm{P}>0.05$ ) (Fig. 1D-F). These suggested that sevoflurane exposure at postnatal days 7-9 dose-dependently impaired the learning and memory of rats after they grew up.

RhoA and ROCK2 upregulation of rat hippocampus is involved in memory dysfunction induced by postnatal exposure of sevoflurane. Corresponding to the behavioral changes of the S3 group, the levels of RhoA, ROCK2 and Cl-Csp3 in the hippocampus of the S3 group significantly increased, compared to that of the $\mathrm{C}$ and $\mathrm{S} 1$ groups $(\mathrm{P}<0.05)$ (Fig. 2 A-C). The ROCK2 inhibitor, fasudil hydrochloride, in the F group clearly decreased the expression of ROCK 2 and Cl-Csp3, and shortened the latency in the training $(\mathrm{P}<0.05$, $\mathrm{P} 46-49$ respectively) and probe test $(\mathrm{P}<0.05)$, compared to that of the S3 group. In addition, there was no significant difference in the expression of ROCK2 and Cl-Csp3, and the latency in the place navigation test and probe test between the $\mathrm{C}$ and $\mathrm{F}$ groups $(\mathrm{P}>0.05)$. These suggested that the increasing expression of RhoA and ROCK2 in the hippocampus was an important mechanism underlying the cognitive dysfunctions induced by sevoflurane exposure. 
A

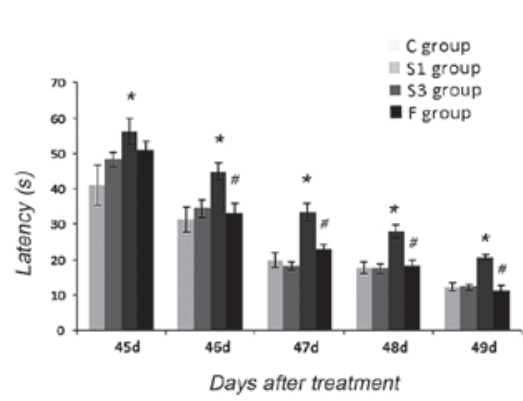

D

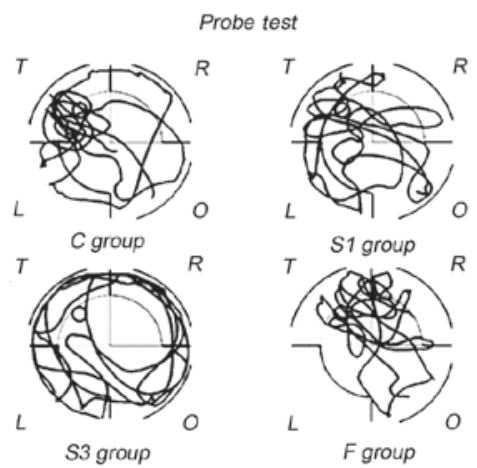

B

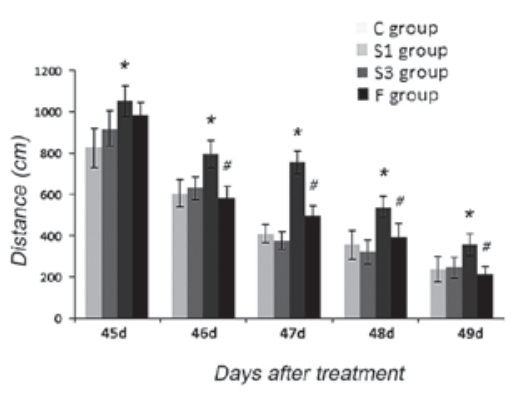

$\mathbf{E}$

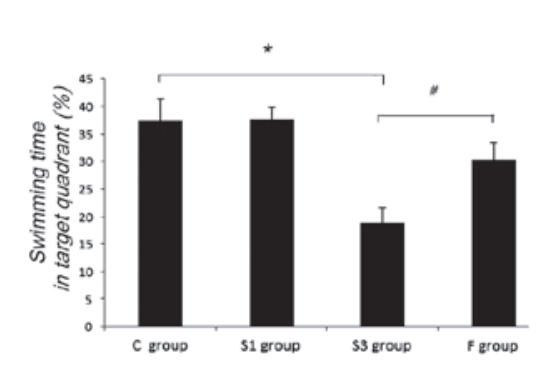

C

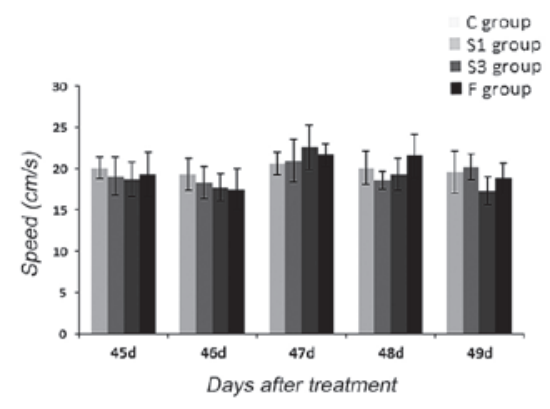

$\mathbf{F}$

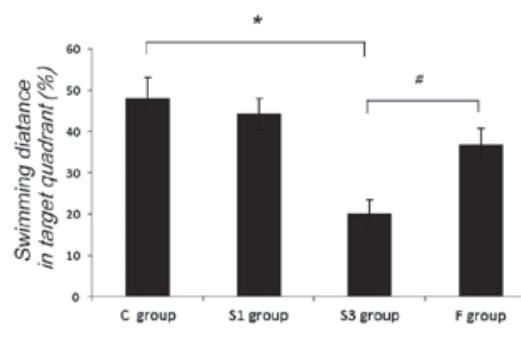

Figure 1. Sevoflurane exposure at postnatal days 7-9 impaired the learning and memory of P45-50 rats in the Morris water maze (MWM) test. (A) In the place navigation test, the latency of the $\mathrm{S} 3$ group was longer than that of the $\mathrm{C}$ and $\mathrm{F}$ groups ( ${ }^{*} \mathrm{P}<0.05, \mathrm{C}$ vs. $\mathrm{S} 3$ group at $\mathrm{P} 45-49$; ${ }^{*} \mathrm{P}<0.05$, $\mathrm{S} 3$ vs. F group at $\mathrm{P} 46-49$ ). There was no significant difference of latency between the $\mathrm{C}$ and $\mathrm{S} 1$ groups $(\mathrm{P}>0.05$, respectively). (B) The distance of the $\mathrm{S} 3$ group was longer than that of the $\mathrm{C}$ and $\mathrm{F}$ groups $\left(" \mathrm{P}<0.05, \mathrm{C}\right.$ vs. S3 group at days $45-49 ;{ }^{\prime \prime} \mathrm{P}<0.05, \mathrm{~S} 3$ vs. F group at $\left.\mathrm{P} 46-49\right)$. There was no significant difference of the distance between the $\mathrm{C}$ and $\mathrm{S} 1$ groups $(\mathrm{P}>0.05$, respectively). (C) The swimming speed had no difference in all the groups $(\mathrm{P}>0.05)$. (D) Representative routes of the $\mathrm{C}, \mathrm{S} 1, \mathrm{~S} 3$ and $\mathrm{F}$ groups in the probe test on P50. T: target quadrant; R, O, L quadrants: right, opposite or left of the target quadrant. (E) In the probe trial test, the percentage of searching time in the target quadrant of the $\mathrm{S} 3$ group was clearly less than that of the $\mathrm{C}$ and $\mathrm{F}$ groups $\left(" \mathrm{P}<0.05, \mathrm{C}\right.$ vs. S3 group; ${ }^{*} \mathrm{P}<0.05, \mathrm{~S} 3$ vs. F group). The percentage of searching time in the target quadrant of the $\mathrm{S} 1$ group was not different from that of the $\mathrm{C}$ group $(\mathrm{P}>0.05)$. ( $\mathrm{F})$ In the probe trial test, the percentage of searching distance in the target quadrant of the $\mathrm{S} 3$ group was clearly less than that of the $\mathrm{C}$ and $\mathrm{F}$ groups ( $\mathrm{P}<0.05, \mathrm{C}$ vs. S3 group; ${ }^{\#} \mathrm{P}<0.05$, S3 vs. F group). The percentage of searching distance in the target quadrant of the $\mathrm{S} 1$ group was nott different from that of the $\mathrm{C}$ group $(\mathrm{P}>0.05)$. Data are expressed as mean \pm standard error of the mean.

A

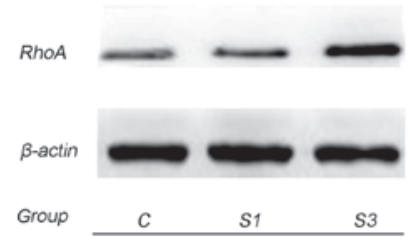

B

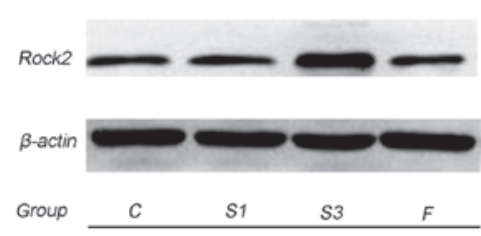

C

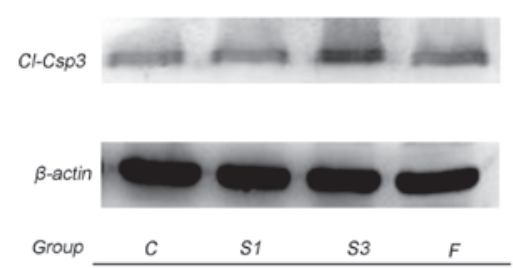

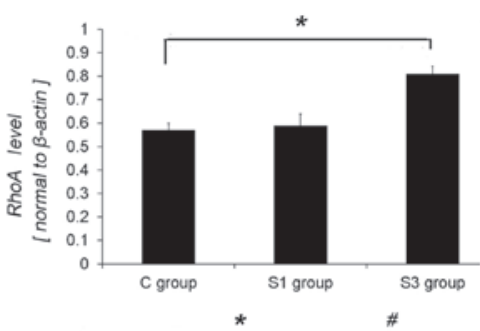
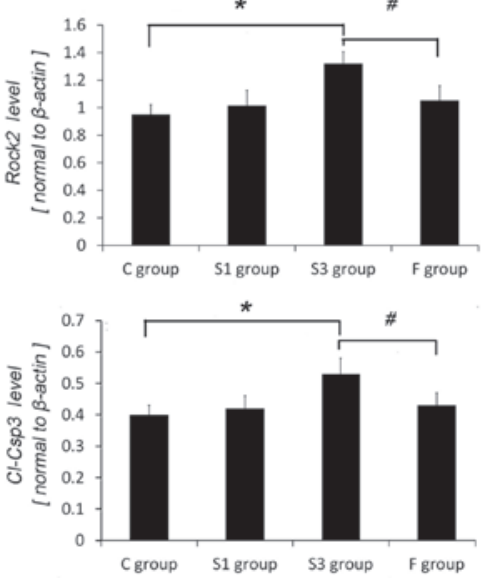

Figure 2. RhoA and ROCK2 upregulation in the rat hippocampus was involved in memory dysfunction induced by postnatal exposure of sevoflurane. The expression of RhoA, ROCK2 and cleaved caspase-3 (Cl-Csp3) in the hippocampus of the S3 group significantly increased, compared to that of the $\mathrm{C}$ group $(\mathrm{P}<0.05)$. There was no difference of the level of RhoA, ROCK2 and $\mathrm{Cl}-\mathrm{Csp} 3$ between the $\mathrm{C}$ and $\mathrm{S} 1$ groups $(\mathrm{P}>0.05)$. ROCK2 inhibitor fasudil hydrochloride in the $\mathrm{F}$ group clearly decreased the expression of ROCK2 and $\mathrm{Cl}-\mathrm{Csp} 3$ ( $\mathrm{P}<0.05$ ), compared to that of the $\mathrm{S} 3$ group ("P<0.05, C vs. $\mathrm{S} 3$ group; ${ }^{\#} \mathrm{P}<0.05, \mathrm{~S} 3$ vs. F group). Data are mean \pm standard deviation. 


\section{Discussion}

The aim of the present study was to investigate whether ROCK2 played roles in the cognitive dysfunction of adult rats exposed to sevoflurane during the postnatal early stage. Sevoflurane exposure during the postnatal early stage dose-dependently impaired the cognitive function of rats after they grew up. Corresponding to the cognitive dysfunction of rats, the level of RhoA, ROCK2 and Cl-Csp3 in the hippocampus in the sevoflurane-treated group significantly increased, compared to the control group. Additionally, ROCK2 inhibitor fasudil hydrochloride decreased the level of ROCK2 and Cl-Csp3 in the hippocampus and partly reversed the cognitive dysfunction in the sevoflurane-treated rats. These showed that ROCK2 was closely involved in the neurotoxicity of sevoflurane.

Neurotoxicity of anesthetics was well reported in aged animals (13-15), natal animals (15) and cultured neurons (16). Yan et al (17) showed that the isoflurane-induced decrease of nNOS is closely correlated with the cognitive impairment in aged rats. In another study, Zheng et al (18) found that sevoflurane use during pregnancy may produce adverse effects on fetal and postnatal rats. In the present study, $2 \%$ sevoflurane for $2 \mathrm{~h}$ at postnatal day 7 did not impair the cognitive function of rats when they grew up. By contrast, 2\% sevoflurane for $2 \mathrm{~h}$ at postnatal days 7-9 ( $2 \mathrm{~h}$ sevoflurane/day) significantly impaired the cognitive function of rats when they grew up. These results showed the dose-dependent neurotoxicity of sevoflurane to the neurons. Consistent with our data, Feng et al (19) found that inhaling $2.3 \%$ sevoflurane for $6 \mathrm{~h}$ induced nerve cell death in newborn rats. Shen et al (20) found that rats in the growing stage (6 days of age) that inhaled $3 \%$ sevoflurane for $2 \mathrm{~h}$ at a time did not show cognitive impairment and nerve inflammation, while those that inhaled 3\% sevoflurane for $2 \mathrm{~h}$ for three days showed cognitive impairment and nerve inflammation. Of note in the present study, corresponding to dose-dependent neurotoxicity of sevoflurane to rats, $2 \%$ sevoflurane of $2 \mathrm{~h}$ at postnatal day 7 did not induce clear expression changes of hippocampal RhoA, ROCK 2 and $\mathrm{Cl}-\mathrm{Csp} 3$, but $2 \%$ sevoflurane of $2 \mathrm{~h}$ at postnatal days 7-9 ( $2 \mathrm{~h}$ sevoflurane/day) significantly increased the expression of hippocampal RhoA, ROCK2 and $\mathrm{Cl}-\mathrm{Csp} 3$. Additionally, when the expression of ROCK2 was inhibited by fasudil hydrochloride, the expression of rat hippocampal ROCK2 and Cl-Csp3 decreased and rat cognitive dysfunction induced by sevoflurane exposure also partly reversed. These showed the key roles of RhoA and ROCK2 in sevoflurane neurotoxicity to natal rats. A previous study showed that RhoA and ROCK2 regulated synaptic plasticity $(6,21)$. Therefore, in the present study, we speculate that sevoflurane may upregulate RhoA and ROCK2, damage synaptic plasticity, and subsequently, induce impairment of learning and memory performance of rats after they grew up. However, its specific mechanism remains to be proved.

\section{Acknowledgements}

The present study was supported by the Hunan Provincial Natural Science Foundation of China (grant no. 12JJ3110) and the 125 Program of The Third Xiangya Hospital, Central South University (Changsha, China).

\section{References}

1. McCann ME, Bellinger DC, Davidson AJ and Soriano SG: Clinical research approaches to studying pediatric anesthetic neurotoxicity. Neurotoxicology 30: 766-771, 2009.

2. Wilder RT, Flick RP, Sprung J, Katusic SK, Barbaresi WJ, Mickelson C, Gleich SJ, Schroeder DR, Weaver AL and Warner DO: Early exposure to anesthesia and learning disabilities in a population-based birth cohort. Anesthesiology 110: 796-804, 2009.

3. Sun L: Early childhood general anaesthesia exposure and neurocognitive development. Br J Anaesth 105 (Suppl 1): i61-i68, 2010.

4. Kalkman CJ, Peelen L, Moons KG, Veenhuizen M, Bruens M, Sinnema G and de Jong TP: Behavior and development in children and age at the time of first anesthetic exposure. Anesthesiology 110: 805-812, 2009.

5. Yon JH, Daniel-Johnson J, Carter LB and Jevtovic-Todorovic V: Anesthesia induces neuronal cell death in the developing rat brain via the intrinsic and extrinsic apoptotic pathways. Neuroscience 135: 815-827, 2005.

6. Wettschureck N and Offermanns S: Rho/Rho-kinase mediated signaling in physiology and pathophysiology. J Mol Med Berl 80: 629-638, 2002 .

7. Negishi $\mathrm{M}$ and Katoh $\mathrm{H}$ : Rho family GTPases as key regulators for neuronal network formation. J Biochem 132: 157-166, 2002.

8. Leong SY, Faux CH, Turbic A, Dixon KJ and Turnley AM: The Rho kinase pathway regulates mouse adult neural precursor cell migration. Stem Cells 29: 332-343, 2011.

9. Sung JK, Miao L, Calvert JW, Huang L, Louis Harkey H and Zhang JH: A possible role of RhoA/Rho-kinase in experimental spinal cord injury in rat. Brain Res 959: 29-38, 2003.

10. Nakazawa T, Watabe AM, Tezuka T, Yoshida Y, Yokoyama K, Umemori H, Inoue A, Okabe S, Manabe T and Yamamoto T: p250GAP, a novel brain-enriched GTPase-activating protein for Rho family GTPases, is involved in the N-methyl-d-aspartate receptor signaling. Mol Biol Cell 14: 2921-2934, 2003.

11. Lemkuil BP, Head BP, Pearn ML, Patel HH, Drummond JC and Patel PM: Isoflurane neurotoxicity is mediated by $\mathrm{p} 75 \mathrm{NTR}-\mathrm{RhoA}$ activation and actin depolymerization. Anesthesiology 114: 49-57, 2011.

12. Pearn ML, Hu Y, Niesman IR, Patel HH, Drummond JC, Roth DM, Akassoglou K, Patel PM and Head BP: Propofol neurotoxicity is mediated by $\mathrm{p} 75$ neurotrophin receptor activation. Anesthesiology 116: 352-361, 2012.

13. He HJ, Wang Y, Le Y, Duan KM, Yan XB, Liao Q, Liao Y, Tong JB, Terrando N and Ouyang W: Surgery upregulates high mobility group box-1 and disrupts the blood-brain barrier causing cognitive dysfunction in aged rats. CNS Neurosci Ther 18: 994-1002, 2012.

14. Stratmann G, Sall JW, May LD, et al: Isoflurane differentially affects neurogenesis and long-term neurocognitive function in 60-day-old and 7-day-old rats. Anesthesiology 110: 834-848, 2009.

15. Shen X, Liu Y, Xu S, Zhao Q, Guo X, Shen R and Wang F: Early life exposure to sevoflurane impairs adulthood spatial memory in the rat. Neurotoxicology 39: 45-56, 2013.

16. Wang WY, Luo Y, Jia LJ, et al: Inhibition of aberrant cyclin-dependent kinase 5 activity attenuates isoflurane neurotoxicity in the developing brain. Neuropharmacology 77: 90-99, 2014.

17. Yan XB, Ouyang W, Li G and Duan KM: Involvement of neuronal nitric oxide synthase in cognitive impairment in isoflurane-treated rats. Neurosci Lett 506: 240-244, 2012.

18. Zheng H, Dong Y, Xu Z, Crosby G, Culley DJ, Zhang Y and Xie Z: Sevoflurane anesthesia in pregnant mice induces neurotoxicity in fetal and offspring mice. Anesthesiology 118: 516-526, 2013.

19. Feng X, Liu JJ, Zhou X, Song FH, Yang XY, Chen XS, Huang WQ, Zhou LH and Ye JH: Single sevoflurane exposure decreases neuronal nitric oxide synthase levels in the hippocampus of developing rats. Br J Anaesth 109: 225-233, 2012.

20. Shen X, Dong Y, Xu Z, Wang H, Miao C, Soriano SG, Sun D, Baxter MG, Zhang Y and Xie Z: Selective anesthesia-induced neuroinflammation in developing mouse brain and cognitive impairment. Anesthesiology 118: 502-515, 2013.

21. Nakano-Kobayashi A, Kasri NN, Newey SE and Van Aelst L: The Rho-linked mental retardation protein OPHN1 controls synaptic vesicle endocytosis via endophilin A1. Curr Biol 19: $1133-1139,2009$. 